Vilmos Prokaj, Department of Probability and Statistics, Eötvös Loránd University, Pázmány Péter sétány 1/C, Budapest, 1117, Hungary. email:

prokaj@cs.elte.hu

\title{
ON A CONSTRUCTION OF J. TKADLEC CONCERNING $\sigma$-POROUS SETS
}

\begin{abstract}
In the paper [2] Josef Tkadlec gave an example of a finite singular Borel measure $\mu$ on the real line such that all $\sigma$-porous sets are of $\mu$ measure zero. We give an alternative proof, i.e. probably a simpler construction, of his theorem $[2$, theorem $]$ and we also give a similar example in $\mathbb{R}^{n}$.
\end{abstract}

A subset $S$ of a metric space $(X, d)$ is said to be porous at a point $x$,

$$
\limsup _{\varepsilon \rightarrow 0} \frac{f(x, \varepsilon)}{\varepsilon}>0
$$

where $f(x, \varepsilon)=\sup \{r: \exists p, B(p, r) \subset B(x, \varepsilon) \backslash S\}$ and $B(p, r)$ denotes the open ball around $p$ with radius $r$. The set $S$ is porous if it is porous at each of its points.

Any porous set $S$ in $\mathbb{R}^{n}$ is of Lebesgue measure zero, because its density is smaller then 1 at any point of $S$. Let $\mu$ be a Borel measure on $[0,1]^{n}$ such that for any $0<c<1$ there exists a constant $d(c)$ depending only on $c$ such that

$$
\frac{\mu(B(p, c r))}{\mu(B(x, r))}>d(c)
$$

provided that $B(p, c r) \subset B(x, r) \subset[0,1]^{n}$, and $r$ is small enough. Then the above argument concerning the $\mu$-density of a porous set $S$ shows that $\mu(S)$ has to be zero.

Key Words: singular measure, $\sigma$-porous set

Mathematical Reviews subject classification: 28A12, 28A35, 28A05

Received by the editors January 15, 2000 
We are going to construct Borel probability measures on $[0,1]^{n}$ satisfying inequality (1), and singular with respect to the Lebesgue measure. It is clear that we can use the "max" distance in $\mathbb{R}^{n}$; i.e.

$$
d_{n}(x, y)=\max _{1 \leq i \leq n}\left|x_{i}-y_{i}\right|
$$

It is enough to show the existence of a singular probability measure on the real line satisfying (2). Indeed if we have such a $\mu$, a singular probability Borel measure on $[0,1]$ then $\mu_{n}=\mu \times \cdots \times \mu$ (n-fold product) is a singular probability measure on $[0,1]^{n}$ such that

$$
\frac{\mu_{n}\left(\times_{i}\left(a_{i}, b_{i}\right)\right)}{\mu_{n}\left(\times_{i}\left(c_{i}, d_{i}\right)\right)}=\prod_{i=1}^{n} \frac{\mu\left(\left(a_{i}, b_{i}\right)\right)}{\mu\left(\left(c_{i}, d_{i}\right)\right)}>d(c)^{n}>0
$$

provided that $\left(a_{i}, b_{i}\right) \subset\left(c_{i}, d_{i}\right) \subset[0,1],\left|b_{i}-a_{i}\right|>c\left|d_{i}-c_{i}\right|$ and $\max \left(\left|d_{i}-c_{i}\right|\right)$ is small enough.

We do something more that is needed. Almost with same effort we can construct a measure such that $d(c)$ can be chosen $d \cdot c$ with $(d=1 / 216)$.

So let $0 \leq \alpha_{n}<1 / 4$ be a decreasing sequence of non-negative numbers. We will denote by $|I|$ the length of the interval $I$. Put

$$
\mu_{n, \alpha}(\{i\})= \begin{cases}\frac{1-\alpha_{n}}{3} & i=0,2 \\ \frac{1+2 \alpha_{n}}{3} & i=1\end{cases}
$$

i.e. we defined a probability measure on the power set of $A=\{0,1,2\}$. Let us form the infinite product measure $\mu_{\alpha}=\prod_{n} \mu_{n, \alpha}$ on the Borel sets of $A^{\mathbb{N}}$. Denote $\nu=\mu_{0}$ the measure we get in this way from the zero sequence.

Define a mapping $T: A^{\mathbb{N}} \rightarrow[0,1]$ by the formula

$$
T(x)=\sum_{k=1}^{\infty} \frac{x(k)}{3^{k}}
$$

i.e. the sequence $x$ is the ternary expansion of $T(x)$. It is well known that there are countable sets $H \subset A^{\mathbb{N}}$ and $H^{\prime} \subset[0,1]$ such that $\left.T\right|_{A^{\mathbb{N}} \backslash H}: A^{\mathbb{N}} \backslash H \rightarrow$ $[0,1] \backslash H^{\prime}$ is bijective, measurable and the inverse is also measurable. So $\mu_{\alpha} \circ T^{-1}$ and $\nu \circ T^{-1}$ are singular if and only if $\mu_{\alpha}$ and $\nu$ is such. Observe that countable sets are of $\mu_{\alpha}$-measure zero, whatever is the sequence $\alpha$ satisfying $0 \leq \alpha_{n}<1 / 4$.

It is also clear that $\nu \circ T^{-1}$ is just the Lebesgue measure on $[0,1]$. An old and famous theorem of Kakutani [1] can be applied to decide which sequences give singular measure $\mu_{\alpha} \circ T^{-1}$ with respect to the Lebesgue measure. He 
introduced the "inner product" of probability measures $\tau, \vartheta$ defined on the same measurable space as follows:

$$
\rho(\tau, \vartheta)=\int\left(\frac{d \tau}{d \pi}\right)^{1 / 2}\left(\frac{d \vartheta}{d \pi}\right)^{1 / 2} d \pi
$$

where both $\tau$, and $\vartheta$ are absolutely continuous with respect to the measure $\pi$. $\rho$ does not depend on the choice of $\pi$. The two measures are the same if $\rho$ is 1 and mutually singular if $\rho$ is 0 .

Theorem 1. ([1, Kakutani 1948]) Let $\mu_{n}, \nu_{n}$ be a sequence of equivalent measures. $\prod_{n} \mu_{n}$ and $\prod_{n} \nu_{n}$ are either singular or equivalent according to

$$
\prod \rho\left(\mu_{n}, \nu_{n}\right)=0 \text { or }>0 \text {. }
$$

Corollary 2. $\mu_{\alpha} \circ T^{-1}$ is singular with respect to the Lebesgue measure if and only if

$$
\sum_{k=1}^{\infty} \alpha_{k}^{2}=\infty
$$

Proof. $\rho\left(\mu_{k, \alpha}, \mu_{k, 0}\right)=1 / 3\left(2 \sqrt{1-\alpha_{k}}+\sqrt{1+2 \alpha_{k}}\right)$ which has logarithm of order $-\alpha_{k}^{2}$.

In what follows let $0<\alpha_{n}<1 / 4$ be a fixed decreasing sequence and $\mu$ denotes $\mu_{\alpha} \circ T^{-1}$. Put

$$
\mathcal{I}_{n}=\left\{\left[\frac{k}{3^{n}}, \frac{k+1}{3^{n}}\right]: k=0,1, \ldots, 3^{n}-1\right\} .
$$

We say that two intervals are joining if one of their endpoints coincide, more precisely $I$ and $J$ are joining if $I \cup J$ is interval and $I \cap J$ has at most one point.

The following two lemmas are trivial corollaries of the definition of $\mu$

Lemma 3. Let $I, J \in \mathcal{I}_{n}$ be two joining intervals, then $1 / 2<\frac{\mu(I)}{\mu(J)}<2$.

Proof. By induction on $n$. For $n=0$ there is nothing to prove. Let $I, J \in$ $\mathcal{I}_{n+1}$ two joining intervals. Either there is $K \in \mathcal{I}_{n}$ such that $I, J \subset K$ or there are joining intervals $K_{0}, K_{1} \in \mathcal{I}_{n}$ such that $I \subset K_{0}$ and $J \subset K_{1}$. In the first case

$$
\frac{1}{2}<\frac{1-\alpha_{n+1}}{1+2 \alpha_{n+1}} \leq \frac{\mu(J)}{\mu(I)} \leq \frac{1+2 \alpha_{n+1}}{1-\alpha_{n+1}}<2
$$


In the second case

$$
\frac{\mu(J)}{\mu(I)}=\frac{\frac{1-\alpha_{n+1}}{3} \mu\left(K_{1}\right)}{\frac{1-\alpha_{n+1}}{3} \mu\left(K_{0}\right)}=\frac{\mu\left(K_{1}\right)}{\mu\left(K_{0}\right)}
$$

which is between $1 / 2$ and 2 by induction hypothesis.

Lemma 4. Let $J \in \mathcal{I}_{n}$ and $I \in \mathcal{I}_{n+k}$ such that $I \subset J$, then

$$
\prod_{l=1}^{k}\left(1-\alpha_{n+l}\right) \leq \frac{3^{k} \mu(I)}{\mu(J)} \leq \prod_{l=1}^{k}\left(1+2 \alpha_{n+l}\right) .
$$

Lemma 5. Let $c>0$ and $I, J$ two subintervals of $[0,1]$ such that $I \subset J$ and $|I|>c|J|$, then

$$
\frac{\mu(I)}{\mu(J)} \geq c \frac{\prod_{l=1}^{m}\left(1-\alpha_{n+l}\right)}{108},
$$

where $n=\left[-\log _{3}(|J|)\right]$ and $m=\left[-\log _{3}(c / 12)\right]+1$ ([a] denotes the integer part of $a$ and $\log _{3}$ stands for the logarithm of base 3.)

Proof. Let $n$ be an integer such that $1 / 3<3^{n}|J| \leq 1$, i.e $n=\left[-\log _{3}(|J|)\right]$. Then there are joining intervals $J_{1}, J_{2} \in \mathcal{I}_{n}$ such that $J \subset J_{1} \cup J_{2}$, e.g. let $J_{1}=[a, b] \in \mathcal{I}_{n}$ such that $(a, b]$ contains the left endpoint of $J$ and let $J_{2}=$ $\left[b, b+3^{-n}\right]$. Therefore

$$
\mu(J) \leq \mu\left(J_{1}\right)+\mu\left(J_{2}\right) \leq 3 \min \left(\mu\left(J_{1}\right), \mu\left(J_{2}\right)\right)
$$

Either for $k=1$ or $k=2$ we have that

$$
\left|I \cap J_{k}\right| \geq \frac{1}{2}|I| \geq \frac{c}{2}|J| \geq \frac{c}{2} \frac{\left|J_{k}\right|}{3}
$$

Let $m$ be an integer such that $3^{-m}<\frac{c}{12} \leq 3 \cdot 3^{-m}$, i.e. $m=\left[-\log _{3}(c / 12)\right]+$ 1 , there is an interval $I_{0}$ in $\mathcal{I}_{n+m}$ such that $I_{0} \subset I \cap J_{k}$ because in $\mathcal{I}_{m+n}$ there are intervals only of length at most the half of the length of $I \cap J_{k}$ and $\cup \mathcal{I}_{n+m}=[0,1]$. So by lemma 4 we get

$$
\mu(I) \geq \mu\left(I_{0}\right) \geq \mu\left(J_{k}\right) 3^{-m} \prod_{l=1}^{m}\left(1-\alpha_{n+l}\right) \geq \mu\left(J_{k}\right) \frac{c}{36} \prod_{l=1}^{m}\left(1-\alpha_{n+l}\right)
$$

Comparing the inequalities (3) and (2) we get the statement.

Corollary 6. Let $0<\alpha_{n}<1 / 4$ be an arbitrary sequence such that 
1. $\alpha_{n} \rightarrow 0$

2. $\sum_{n} \alpha_{n}^{2}=\infty$.

The associated Borel measure $\mu_{\alpha} \circ T^{-1}$ is singular and takes on a value of zero on any $\sigma$-porous Borel set.

Proof. By property 1 for any $c>0$ there is an $n_{0}$ such that for $n \geq n_{0}$, $\prod_{l=1}^{m}\left(1-\alpha_{n+l}\right)>1 / 2$ where $m=\left[-\log _{3}(c / 12)\right]+1$. This means that if $J$ is small enough and $I \subset J,|I|>c|J|$ then

$$
\frac{\mu(I)}{\mu(J)}>\frac{c}{216}
$$

So the sufficient condition (1) is satisfied, $\mu$ assigns measure zero to porous sets. By the theorem of Kakutani this measure is singular with respect to the Lebesgue measure.

Corollary 7. There is a continuum family of pairwise mutually singular Borel measures on $[0,1]$ such that each measure is singular with respect to the Lebesgue measure and takes on a value of zero on $\sigma$-porous sets.

Proof. Let $\mu_{a}$ be the measure corresponding to $\alpha_{n}=a \cdot n^{-1 / 2}$ where $a \in$ $(0,1 / 4)$. Using the previous corollary we have to prove only that if $a, b \in$ $(0,1 / 4)$ and $a \neq b$ then $\mu_{a}$ and $\mu_{b}$ are mutually singular. We can apply Kakutani's theorem again since

$$
\rho\left(\mu_{n, a}, \mu_{n, b}\right)=\frac{2}{3} \sqrt{\left(1-\frac{a}{\sqrt{n}}\right)\left(1-\frac{b}{\sqrt{n}}\right)}+\frac{1}{3} \sqrt{\left(1+2 \frac{a}{\sqrt{n}}\right)\left(1+2 \frac{b}{\sqrt{n}}\right)}
$$

which has logarithm of order $-n^{-1}$.

The above construction gives something more that we actually need. Indeed for the constructed measure the constant $d(c)$ can depend on $c$ linearly. If we do not care about this we can choose $0<\alpha_{n}<1 / 4$ to be a constant sequence and then instead of Kakutani's theorem we can apply the strong law of large numbers to see that $\mu_{\alpha}$ is singular with respect to the Lebesgue measure.

\section{References}

[1] Shizuo Kakutani, On equivalence of infinite product measures, Ann. of Math. 2(1948), 49:214-224. 
[2] Josef Tkadlec, Construction of a finite Borel measure with $\sigma$-porous sets as null sets, Real Anal. Exchange, 12(1)(1986/87), 349-353. 